\title{
3D PRINTED DIFFERENTIAL FORCE AND POSITION SENSOR BASED ON LOSSY TRANSMISSION LINES
}

\author{
Martijn Schouten, Parth Patel, Remco Sanders and Gijs Krijnen
}

Robotics And Mechatronics group, University of Twente, Enschede, The Netherlands

\begin{abstract}
This paper describes a $3 \mathrm{D}$ printed differential capacitive sensor, which uses the piezoresistivity of the used electrodes to determine both the force that is applied to the sensor and the position where the force is applied. To do so, both the real and the imaginary parts of the sensor's impedance is used. We present the idea, its analysis as well as experimental results showing the feasibility of our approach.
\end{abstract}

\section{INTRODUCTION}

Previously it has been shown that a flexible parallel plate capacitor can be used to measure the applied normal force [1]. It also has been shown that a similar structure using multifrequency readout can be used to measure force and the position where it is applied in 2D [2]. Structures like these enable flexible devices with few connections while still offering the possibility to determine a $2 \mathrm{D}$ resolved position of the point of contact of a force.

In a comparable fashion, we here introduce a method to measure the magnitude of a force as well as its position along a given elongated structure by measuring the real and imaginary impedance of a 3D printed differential capacitive structure at a single frequency below the cut-off frequency of the sensor. In this approach, the imaginary part of the impedance exclusively gives information on the magnitude of the force whereas the real part is affected by both the magnitude and the position of the force through the piezoresistive effect of the material. By doing so we show that it is already possible to measure the position where the force is applied using a single frequency measurement.

\section{OPERATING PRINCIPLE AND MODEL}

A cross-section of the proposed structure is shown in figure 1. The basis of the structure is formed by 3 layers of conductive thermoplastic polyurethane (TPU) layers, acting as the electrodes of the two capacitors. Between the top and middle layer, a soft layer of X60 of 70\% infill provides a relative deformable upper capacitor, whereas the X60 layer of $100 \%$ infill between the middle and bottom electrode provides a much stiffer structure, to act as reference capacitance. A circuit representation of an infinitely small part of the proposed geometry is shown in figure 2

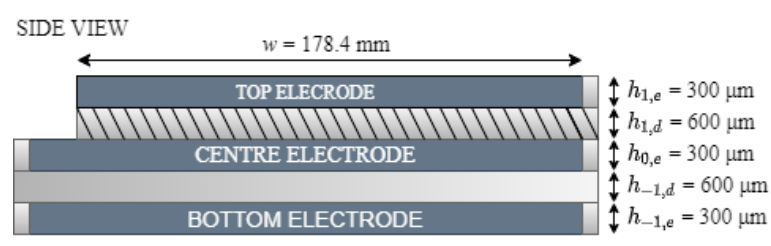

$\mathrm{X} 60(100 \%$ infill $) \quad \mathrm{X} 60$ (70\% infill) $\square$ PI-ETPU $85-700+$

Fig. 1. Dimension of the 3D printed sensor.

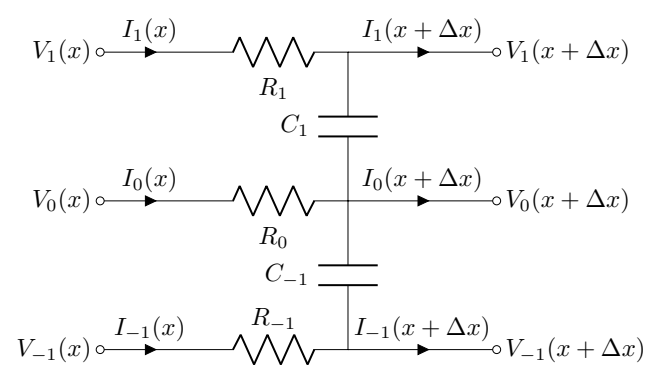

Fig. 2. Electrical circuit diagram of an infinitesimal part of the sensor

Due to the large resistance of the parallel plates of the $3 \mathrm{D}$ printed parallel plate capacitors they are expected to behave like a very lossy transmission line. The differential capacitive sensor described in this work uses two of these parallel plate capacitors printed on top of each other in order to compensate for drift and non-linearity in mainly the resistive part. The sensor therefore can be seen as a combination of two coupled lossy transmission lines, as illustrated by figure 1 .

The behaviour of the voltage along the different electrodes in this circuit can be described with the following differential equations $[3$, p. 50$]$ :

$$
\frac{d V_{n}(x)}{d x}=-Z_{n} I_{n}(z)
$$

The current can be described using

$$
\begin{aligned}
\frac{d I_{1}(x)}{d x} & =G_{1}\left(V_{0}(x)-V_{1}(x)\right) \\
\frac{d I_{0}(x)}{d x} & =G_{1}\left(V_{1}(x)-V_{0}(x)\right)+G_{-1}\left(\left(V_{-1}(x)-V_{0}(x)\right)\right. \\
\frac{d I_{-1}(x)}{d x} & =G_{-1}\left(V_{0}(x)-V_{-1}(x)\right)
\end{aligned}
$$

with $R$ the resistance per meter length of the electrodes and $G_{n}$ the admittance per meter length of the dielectric. Both are depending on the material properties and the geometry of the sensor through:

$$
\begin{aligned}
Z_{n} & =\frac{\rho_{n}}{h_{n, e} w} \\
G_{n} & =j \omega C_{n}=\frac{j \omega \epsilon_{n} w}{h_{n, d}}
\end{aligned}
$$

Where $\epsilon_{n}$ and $\rho_{n}$ are the relative permittivity of the dielectrics and the resistivity of the electrodes respectively. The geometry constants $h_{e}, w$ and $h_{n}$ are defined in Figure 1. To solve this system of equations it is rewritten into matrix form:

$$
\frac{d \vec{S}}{d x}=\mathbf{A} \vec{S}
$$




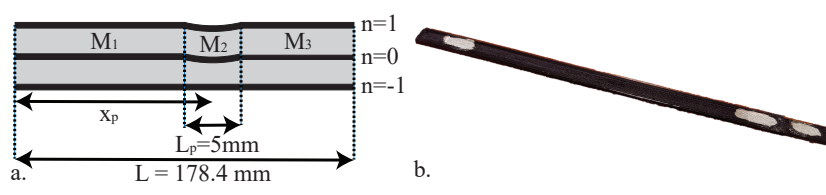

Fig. 3. a. Definition of the different sections of the sensor. b. A picture of the sensor

With:

$$
S=\left[\begin{array}{c}
V_{1} \\
I_{1} \\
V_{0} \\
I_{0} \\
V_{-1} \\
I_{-1}
\end{array}\right], A=\left[\begin{array}{cccccc}
0 & -Z_{1} & 0 & 0 & 0 & 0 \\
-G_{1} & 0 & G_{1} & 0 & 0 & 0 \\
0 & 0 & 0 & -Z_{0} & 0 & 0 \\
G_{1} & 0 & -G_{1}-G_{-1} & 0 & G_{-1} & 0 \\
0 & 0 & 0 & 0 & 0 & -Z_{-1} \\
0 & 0 & G_{-1} & 0 & -G_{-1} & 0
\end{array}\right]
$$

The form of equation 4 suggests that a solution of this set of equations can be found from exponential functions based on the eigenvalues and corresponding eigenvectors, subjected to the proper boundary conditions. Or:

$$
\vec{S}(x)=\mathbf{Y}(x) \vec{C}
$$

with $\mathbf{Y}(x)$ a matrix formed by the eigenvectors $\vec{V}_{n}$ and the eigenvalues $\lambda_{n}$.

$$
\mathbf{Y}(x)=\left[\begin{array}{llll}
\vec{V}_{1} e^{\lambda_{1} x} & \vec{V}_{2} e^{\lambda_{2} x} & \ldots & \vec{V}_{6} e^{\lambda_{6} x}
\end{array}\right]
$$

and $\vec{C}$ a vector of coefficients determined by the boundary conditions. In case $\vec{S}(x)$ is known at a certain position $x$, $\vec{C}$ can be obtained using:

$$
\vec{C}=\mathbf{Y}^{-1}(x) \vec{S}(x)
$$

This can be used to calculate the propagation of a known $S(0)$ over a length $L$ using.

$$
\vec{S}(L)=\mathbf{N}(L) \vec{S}(0)
$$

with:

$$
\mathbf{N}(L)=\mathbf{Y}(L) \mathbf{Y}^{-1}(0)
$$

The sensor will have slightly different parameters at the position where it is pressed compared to the positions where there is no applied pressure. Therefore in the analysis, we split the sensor into three parts, see figure 3. Each of these sections will have its own $\mathbf{N}$ matrix, calculated using different parameters. Because the output of one section is connected to the next section's input, the overall behavior can be obtained by multiplication of the respective $\mathbf{N}$ matrices. The matrix that can be used to calculate the propagation through the different sections in case $\vec{S}(0)$ is known, as in equation 9, can be calculated as follows.

$\mathbf{N}_{\text {tot }}(L)=\mathbf{N}_{3}\left(L-x_{\mathrm{p}}-\frac{1}{2} L_{\mathrm{p}}\right) \mathbf{N}_{2}\left(L_{\mathrm{p}}\right) \mathbf{N}_{1}\left(x_{\mathrm{p}}-\frac{1}{2} L_{\mathrm{p}}\right)$

In this work, the measurement setup applies a fixed voltage on the input of the sensor. The other side of the sensor is not connected, and therefore, the current going out is fixed at 0 . In order to be able to apply this mixed boundary condition, masking matrices $\mathbf{M}_{U}$ and $\mathbf{M}_{I}$ are used to mask out the voltage and the current rows. This results in the following boundary condition equation.

$$
\mathbf{M}_{U} \vec{S}(0)+\mathbf{M}_{I} \vec{S}(L)=\vec{B}
$$

TABLE I

Printing parameters

\begin{tabular}{l|cccc} 
& $\mathrm{X} 60$ & $\mathrm{X} 60$ & $\mathrm{PI}-\mathrm{ETPU}$ & $\mathrm{BVOH}$ \\
& $100 \%$ & $70 \%$ & $85-700+$ & \\
\hline Infill & $100 \%$ & $70 \%$ & $100 \%$ & $100 \%$ \\
Wall line count & 3 & 3 & 5 & 5 \\
Top layers & N/A & 1 & N/A & N/A \\
Bottom layers & N/A & 1 & N/A & N/A
\end{tabular}

With:

$$
\mathbf{M}_{U}=\left[\begin{array}{llllll}
1 & 0 & 0 & 0 & 0 & 0 \\
0 & 0 & 0 & 0 & 0 & 0 \\
0 & 0 & 1 & 0 & 0 & 0 \\
0 & 0 & 0 & 0 & 0 & 0 \\
0 & 0 & 0 & 0 & 1 & 0 \\
0 & 0 & 0 & 0 & 0 & 0
\end{array}\right], \mathbf{M}_{I}=\left[\begin{array}{llllll}
0 & 0 & 0 & 0 & 0 & 0 \\
0 & 1 & 0 & 0 & 0 & 0 \\
0 & 0 & 0 & 0 & 0 & 0 \\
0 & 0 & 0 & 1 & 0 & 0 \\
0 & 0 & 0 & 0 & 0 & 0 \\
0 & 0 & 0 & 0 & 0 & 1
\end{array}\right], \vec{B}=\left[\begin{array}{c}
V_{1}(0) \\
I_{1}(L) \\
V_{0}(0) \\
I_{0}(L) \\
V_{-1}(0) \\
I_{-1}(L)
\end{array}\right]
$$

Using these boundary conditions and equation 9 and 11 , the current going into each electrode, which is part of $\vec{S}(0)$, can be calculated.

$$
\vec{S}(0)=\left(\mathbf{M}_{U}+\mathbf{M}_{I} \mathbf{N}_{\text {tot }}\right)^{-1} \vec{B}
$$

Next the impedance measured by the LCR can be calculated using:

$$
Z=-\frac{V_{1}(0)-V_{0}(0)}{I_{0}(0)}
$$

What remains is a prediction of the change in the parameters of section 2. To predict the change in thickness, the following equation can be used [1].

$$
\Delta h_{n, d}=-\frac{F h_{n, d}}{A_{0} E_{n}^{\prime}}
$$

Where $\Delta h$ is the change in thickness, $F$ is the applied force, $h_{n}$ the original thickness, $A_{0}$ the original area and $E^{\prime}$ the effective Young's modulus. Since there is a relatively large amount of air in both dielectrics due to the 3D printing process, they will be assumed compressible (Poisson ratio of zero), and the change in the area of the plates will be neglected.

The piezoresistivity of the electrodes will be modeled on a macroscopic level, with a sensitivity factor $K_{n}$ indicating the relative change in resistivity with applied force.

$$
\frac{\Delta \rho_{n}}{\rho_{n}}=K_{n} F
$$

\section{METHODOLOGY \\ Sensor fabrication}

The sensor was designed in Autodesk Inventor and sliced using Cura 4.7.1, using a custom post-processing script [4] and the parameters in table I. The sensor was printed on a Diabase H-series 3D printer and on top of a $200 \mu \mathrm{m}$ thick layer of BVOH to make it easy to remove the sensor without damaging it. Figure 1 shows the dimensions of the $3 \mathrm{D}$ printed sensor.

\section{Measurement setup}

The sensor is placed on a mount and clamped from both ends. Electrical connections are made by soldering the wires to copper tape on the clamps. The copper tape is clamped onto silver conductive paint (Electrolube SCP26G) painted on the electrodes. The sensor mount is placed on a grounded steel plate and fixed on the bed of a Rova3D printer by ORD solutions. The linear actuator is clamped on a steel frame, 


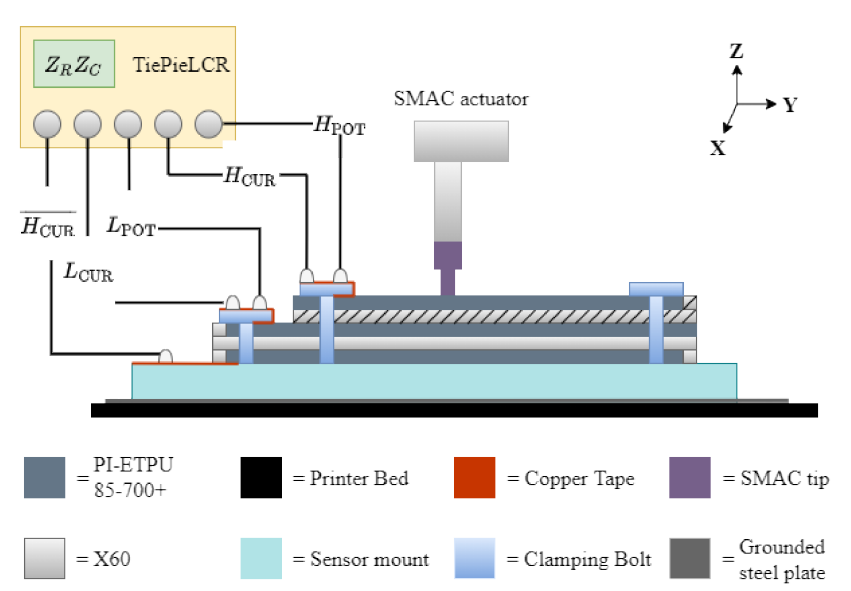

Fig. 4. Schematic of measurement setup

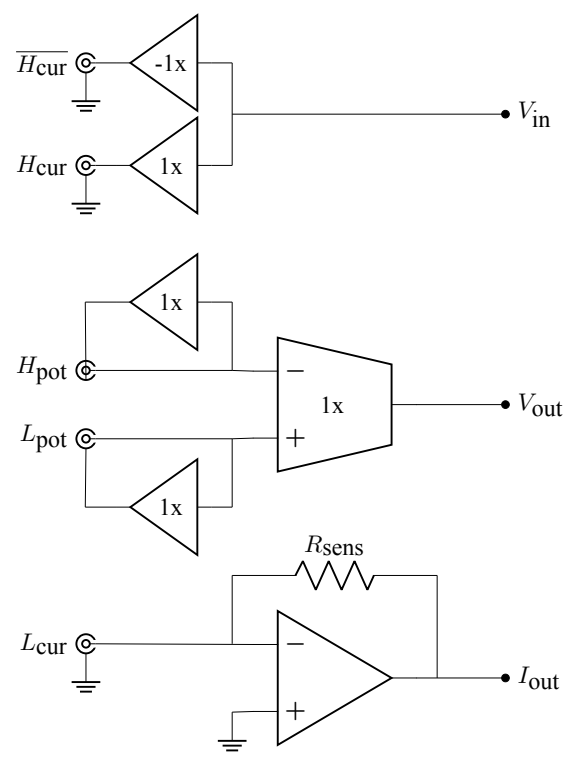

Fig. 5. Simplified circuit of the TiePieLCR. See Figure 4 for the connection of this circuit to the sensor.

fixing it at a fixed height above the print bed. To measure the impedance as a function of both force and position, the linear actuator is set to consecutively apply a compressive force of 4,5,6,9 and $12 \mathrm{~N}$ for 2 seconds and then the measurement is repeated after increasing the $y$ position of the bed.

\section{Sensor readout}

The readout of the sensor is done using a differential auto-balancing bridge circuit [5], [6]. This circuit is build using LTC6268 and LTC6268-10 opamps, which combine a high input impedance with a large gain-bandwidth. The input impedance of the voltage measurement channels, $H_{\text {pot }}$ and $L_{\text {pot }}$, are increased further by guarding the cables [7, p. 359]. The sensor is connected to this circuit as defined in Figure 4. The outputs $V_{\text {out }}$ and $I_{\text {out }}$ are measured using a Handyscope HS5-540 by TiePie Engineering, streaming at $3.125 \mathrm{MS} \mathrm{s}^{-1}$. The harmonic excitation signal is generated using the arbitrary waveform generator of the Handyscope and connected to $V_{\mathrm{in}}$. A python script is used to demodulate the voltage and current in order to calculate the impedance. The advantage of this approach is that the impedance can be measured continuously and with a large bandwidth. A
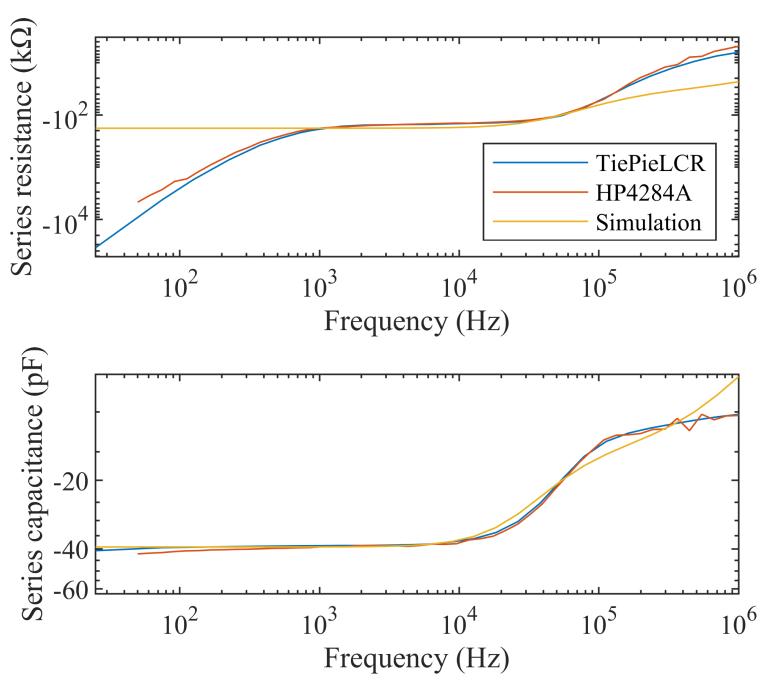

Fig. 6. Measured and predicted differential impedance spectrum

TABLE II

Parameters for fig 7

\begin{tabular}{c|c|c|c|c|c}
$\begin{array}{c}\text { Para- } \\
\text { meter }\end{array}$ & $\begin{array}{c}\text { Fit } \\
\text { Fig 7 }\end{array}$ & $\begin{array}{c}\text { Start } \\
\text { Fig 7 }\end{array}$ & $\begin{array}{c}\text { Fit } \\
\text { Fig 6 }\end{array}$ & $\begin{array}{c}\text { Start } \\
\text { Fig 6 }\end{array}$ & Unit \\
\hline$\epsilon_{1}$ & 4.68 & 4.66 & 4.76 & 5.00 & \\
$\epsilon_{-1}$ & 6.54 & 6.54 & 6.54 & 6.70 & \\
$\rho_{1}$ & 0.16 & 0.15 & 0.21 & 1.00 & $\Omega \mathrm{m}$ \\
$\rho_{0}$ & 0.99 & 0.93 & 1.33 & 1.00 & $\Omega \mathrm{m}$ \\
$\rho_{-1}$ & 0.04 & 0.04 & 0.05 & 1.00 & $\Omega \mathrm{m}$ \\
$E_{1}^{\prime}$ & 0.93 & 1.00 & N.A. & N.A. & $\mathrm{MPa}$ \\
$E_{-1}^{\prime}$ & 6.00 & 6.00 & N.A. & N.A. & $\mathrm{MPa}$ \\
$K_{1}$ & 0.41 & 0.03 & N.A. & N.A. & $\mathrm{N}^{-1}$ \\
$K_{0}$ & 0.03 & 0.03 & N.A. & N.A. & $\mathrm{N}^{-1}$ \\
$K_{-1}$ & 0.67 & 0.03 & N.A. & N.A. & $\mathrm{N}^{-1}$
\end{tabular}

simplified version of the circuit can be found in figure 5 and from here on will be referred to as TiePieLCR.

To verify the impedance spectrum measured by the readout circuitry the spectrum is also measured using a HP4284A LCR meter. To obtain $\overline{H_{\text {cur }}}$ for the differential measurement, the $H_{\text {cur }}$ output of HP4284A is split by connecting the $H_{\text {cur }}$ of the HP4284A to the $V_{\text {in }}$ of the TiePieLCR.

\section{Fitting}

Both the impedance spectrum and measured impedance against force and position are fitted to the model by running Matlab's patternsearch on 6 cores of an i7 $9850 \mathrm{H}$ for $30 \mathrm{~s}$. For the fit of the impedance spectrum, only frequencies between $1 \mathrm{kHz}$ and $500 \mathrm{kHz}$ have been used. Both the starting parameters and the fitted parameters can be found in Table 7.

\section{RESULTS}

Figure 6 shows the measured and the simulated impedance spectrum. Figure 7 shows the simulated capacitance and resistance measurement as a function of the applied force and the position where the force is applied. For these simulations, the fitted parameters in table II were used. The capacitance and the resistance are negative since the differences between the top and bottom capacitor are measured, and the bottom capacitor's capacitance and electrode resistance are larger. The figure shows that the imaginary part is only dependent on the force, while the real part is also dependent on the position. 
Figure 9 shows the measured capacitance and resistance as a function of the applied force and position.

Figure 8 plots the same data as Figure 7, but shows the force and position as a function of the capacitance and resistance, showing that it is possible to determine the position and the force from these two measurements.
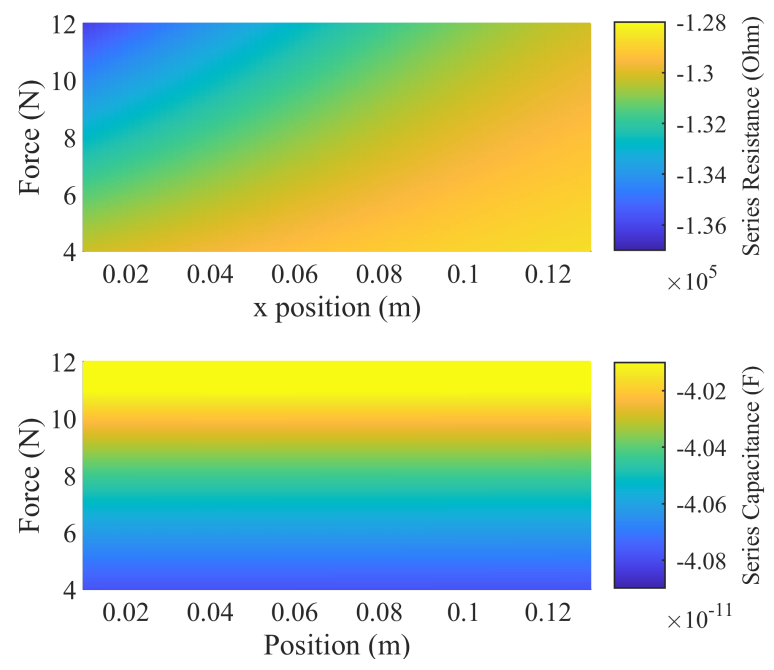

Fig. 7. Differential impedance against force and position as predicted by the model
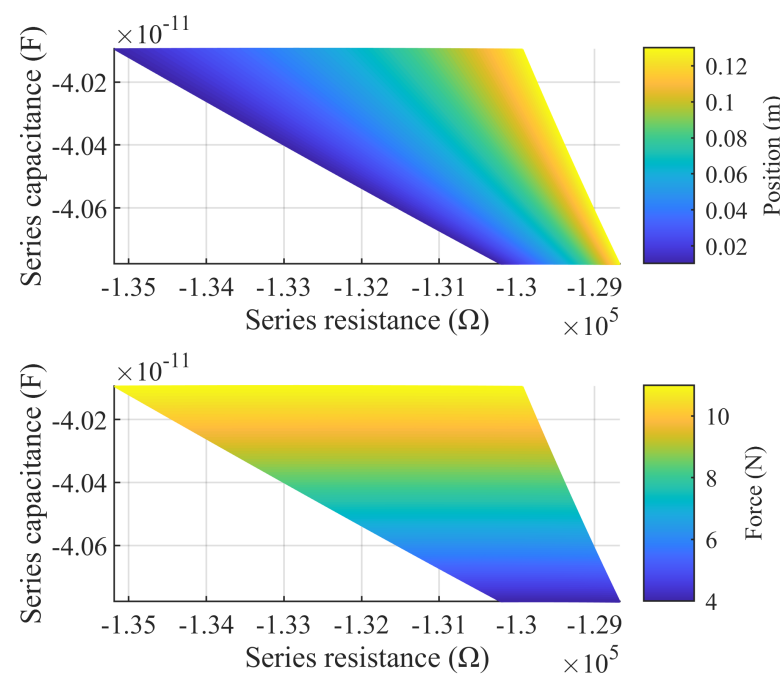

Fig. 8. Force and position against the differential impedance as predicted by the model

\section{CONCLUSION AND DISCUSSION}

In this work we presented a model for a sensor based on a lossy transmission line that can be used to measure both the magnitude and position of an applied force. A sensor was designed using this model and subsequently $3 \mathrm{D}$ printed. The capacitance of this sensor indeed was only dependent on the applied force, while the real part of this sensor indeed showed a dependence on both the position and the applied force.

The measured spectrum deviates from the model at low and high frequencies. The deviation in the resistance at low frequencies is expected to be due to the model not taking into account dielectric loss and leakage of the dielectric.
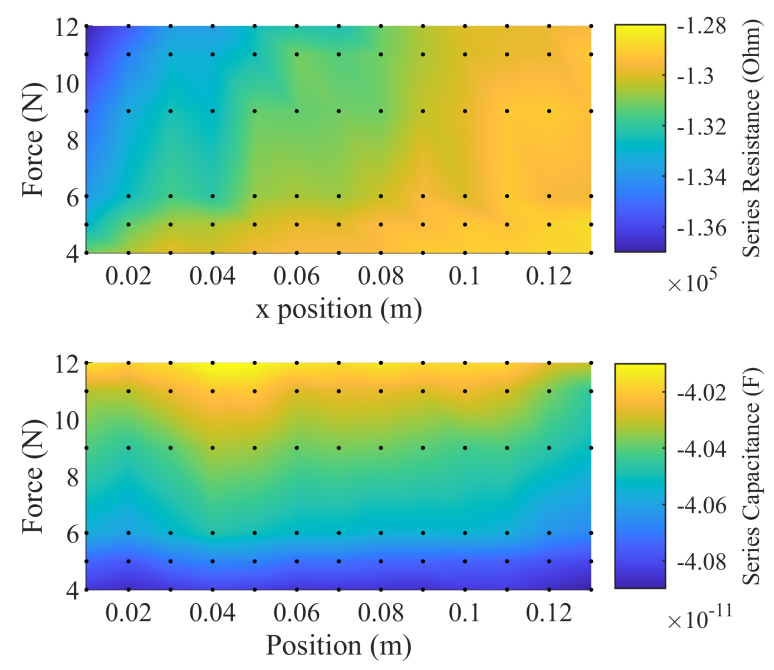

Fig. 9. Measured differential impedance against force and position. The black dots are measurement points.

The deviation at high frequencies might be due to the steel ground plate not being taken into account in the model.

This method has shown that it is possible to obtain even more information from the same structure, which might enable multi-touch applications or increased sensitivity when combined with multi-frequency readout. Although the current method is only implemented in $1 \mathrm{D}$, the method might be implemented in 2D by using a similar method as used by $\mathrm{Xu}$ et al.

\section{REFERENCES}

[1] M. Schouten, R. Sanders, and G. Krijnen, "3D printed flexible capacitive force sensor with a simple microcontroller based readout," Proceedings of IEEE Sensors, vol. 2017-Decem, no. 3, pp. 1-3, 2017.

[2] D. Xu, A. Tairych, and I. A. Anderson, "Stretch not flex: programmable rubber keyboard," Smart Materials and Structures, vol. 25, no. 1, p. 015012, nov 2015. [Online]. Available: https://doi.org/10.1088/0964$1726 / 25 / 1 / 015012$

[3] D. M. Pozar, Microwave engineering, 3rd ed. John Wilen \& Sons, Inc., 2005.

[4] M. Schouten, "diabase cura post processor," 2020. [Online]. Available: https://github.com/ martijnschouten/diabase_cura_post processor

[5] Keysight Technologies, "Impedance measurement handbook," access date: 16-02-2021. [Online]. Available: https://www.keysight.com/nl/en/assets/701806840/application-notes/5950-3000.pdf

[6] A. Dagamseh, C. Bruinink, R. Wiegerink, T. Lammerink, H. Droogendijk, and G. Krijnen, "Interfacing of differential-capacitive biomimetic hair flow-sensors for optimal sensitivity," Journal of micromechanics and microengineering, vol. 23, no. 3, p. 035010 , Mar. 2013.

[7] P. Horowitz and W. Hill, The Art of Electronics, 3rd ed. USA: Cambridge University Press, 2015. 